\title{
CAUSES OF HOSPITALIZATION OF SYSTEMIC LUPUS ERYTHEMATOSUS PATIENTS IN A TERTIARY HOSPITAL
}

Maria Thereza Gomes Caldeira ${ }^{1, \star}$, Leticia Cezar Araújoํㅜ, Fernanda Lourenço Macagnani ${ }^{1}$, Camila Ávila Megda Cabianca ${ }^{1}$, Renê Donizeti Ribeiro De Oliveira ${ }^{1}$

1.Universidade de São Paulo, Ribeirão Preto (SP), Brazil.

*Corresponding author: emailnumero9@gmail.com

\section{BACKGROUND}

Systemic lupus erythematosus (SLE) is a chronic, multisystemic, immune-mediated disorder associated with a substantial hospitalization risk. The studies on frequency and causes of hospitalizations are conflicting. We aim to describe causes and rates of hospitalization for SLE patients in a tertiary reference hospital.

\section{METHODS}

This cross-sectional retrospective study evaluated SLE patients aged 18-65 years and admitted to infirmaries from January 2014 to December 2020. Medical records were revised for demographic, clinical and laboratory characteristics, previous and ongoing treatments and the reason for admission.

\section{RESULTS}

We included 452 patients. The mean age was 37 years, and 88\% were females. Except for 7.5\% newly diagnosed SLE, all patients were in use of immunosuppressive drugs (azathioprine, methotrexate or cyclophosphamide) and glucocorticoids up to $1 \mathrm{mg} / \mathrm{kg} /$ day of prednisone or equivalent. Flare (or active disease) of SLE was the most common cause of hospitalization (44\%), followed by infection (24\%) and treatment with intravenous drugs (16\%). Approximately $3.5 \%$ of patients were admitted for gynecological or obstetric reasons, and $1.5 \%$ for treatment of manifestations related to antiphospholipid antibody syndrome. Other causes contributed for $10 \%$ of admissions. Flares were due to renal (14\%), more than one organ or system (12\%), central and peripheral nervous system (8\%), hematologic (2.5\%), pulmonary (2\%), cardiac (1.5\%), blood vessels (1\%), serosa (1\%), joint (0.9\%) and skin ( $0.6 \%)$ involvement.

\section{CONCLUSION}

Flare was the most common reason for hospital admission in SLE, which matches previously published studies. Kidneys were the most frequently affected organ, which is consistent with previous reports. Infection was the second most frequent cause of hospitalization, and may be related to the disease itself, associated comorbidities or immunosuppressive therapy. Manifestations of the disease were the main cause of hospitalizations in SLE. Infections presented as another important cause of need for hospital care, which can be at least in part attributed to immunosuppressive therapy.

\section{KEYWORDS}

Systemic lupus erythematosus patients, Hospitalization, Prevalence. 